\title{
EXTENDED COHOMOLOGICAL FIELD THEORIES AND NONCOMMUTATIVE FROBENIUS MANIFOLDS
}

\author{
S.M. NATANZON
}

\author{
Independent University of Moscow, Moscow, Russia \\ Moscow State University, Moscow, Russia \\ Institute Theoretical and Experimental Physics, Moscow, Russia
}

\begin{abstract}
We construct some extension (Stable Field Theory) of Cohomological Field Theory. The Stable Field Theory is a system of homomorphisms to some vector spaces generated by spheres and disks with punctures. It is described by a formal tensor series, satisfying to some system of "differential equations". In points of convergence the tensor series generate special noncommutative analogues of Frobenius algebras, describing 'Open-Closed' Topological Field Theories.

MSC: $81 \mathrm{~T}, 18 \mathrm{~F}$

Subj. Clac. field theory

Keywords: Field theory; Frobenius manifold
\end{abstract}

\section{INTRODUCTION}

Cohomological Field Theories were proposed by Kontsevich and Manin [7] for description of Gromov-Witten Classes. They proved that Cohomological Field Theory is equivalent to Formal Frobenius manifold. Formal Frobenius manifold is defined by a formal series $F$, satisfying the associative equations [5, 14. In points of convergence the series $F$ defines Frobenius algebras. The set of these points forms a Frobenius manifold as regards to algebras investigated (with some additional condition) by Dubrovin [6].

Cohomological Field Theory is a system of special homomorphisms to spaces of cohomology of Deline-Mumford compactifications for moduli spaces of complex rational curves (Riemann spheres) with punctures. In this paper it is constructed some extension of Cohomological Field Theory. This extension (Stable Field Theory) is a system of homomorphisms to some vector spaces generated by disks with punctures. I conjecture that they describe relative Gromov-Witten classes.

A Stable Field Theory is equivalent to some analog of a Formal Frobenius manifold. This analogue is defined by formal tensor series (Structure Series), satisfying some system of "differential equations" 
(including the associativity equation). In points of convergence the Structure Series define Extended Frobenius algebras. They are special noncommutative analogue of Frobenius algebras. Extended Frobenius algebras describe 'Open-Closed' Topological Field Theories [8] of genus 0 in the same way as Frobenius algebras describe Atiyah-Witten $2 D$ Topological Field Theories [2, 13]. Thus, Structure Series are noncommutable analogues of Formal Frobenius manifolds.

In sections 1 and 2 it is proposed a general axiomatic of Topological Field Theories over functors. This class involves $2 D$ Atiyah-Witten [2, 13, 'open-closed' [8, Klein Topological Field Theories [3] and Cohomological Field Theories [7, 9].

In section 3 it is constructed and investigated a Stabilising functor on a category of spheres with punctures, disks with punctures and its disconnected unions. For spheres this construction is liked to modular graphs [9] and describes a Cohomological functor for complex rational curves. The Stable Field Theories are defined as Topological Field Theories over Stabilizing functors.

In section 4 and 5 we prove that Stable Field Theories are in oneto-one correspondence to Systems of Disk Correlation Functions. Its "generating functions" are the Structure Series

In section 6 and 7 it is demonstrated that Structure Series generate Extended Frobenius algebras in their convergence points and it is constructed some examples.

A part of this paper was written during the author stays at Erwin Schrödinger Institute in Vena, Max-Plank-Institut in Bonn and UNED in Madrid. I thank these organisations for support and hospitality. I would like to thank B.Dubrovin and S.Novikov for useful discussions of results. This research is partially supported by grants RFBR-02-0122004a, NSh-1972.2003.1.

\section{1. $2 D$ CATEGORIES}

1.1. In this paper a surface denotes a compact surface with or without boundary. Its connected boundary component is called a boundary contour of the surface. Any orientable connected surface is homeomorphic to a sphere with $g$ handles and $s$ holes. Such surface is called $a$ surface of type $(g, s, 1)$. Any nonorientable connected surface is homeomorphic to either a projective plane with $a$ handles and $s$ holes or a Klein bottle with $a$ handles and $s$ holes. Such surface is called a surface of type $(g, s, 0)$, where $g=a+\frac{1}{2}$ in the first case and $g=a+1$ in the second case.

A surface with a finite number of marked points is called a stratified surface. Marked points are also called special points of the stratified surface. Two stratified surfaces are called isomorphic, if there exists a homeomorphism of the surfaces, generating a bijection between their special points. 
By $|S|$ we denote the cardinality of a finite set $S$. Let $\Omega$ be a surface of the type $(g, s, \epsilon)$ and $\omega_{1}, \ldots, \omega_{s}$ be its boundary contours. Let $S \subset \Omega$ be a finite set of marked points and $m=|S \cap(\Omega \backslash \partial \Omega)|, m_{i}=\left|S \cap \omega_{i}\right|$. Then the collection $G=\left(g, \epsilon, m, m_{1}, \ldots, m_{s}\right)$ is called a type of connected stratified surface $(\Omega, S)$. A connected stratified surface of type $G$ is called trivial, if $\mu=2 g+m+s+\frac{1}{2} \sum m_{i}-2 \leqslant 0$. It is easy to prove the following statement.

Lemma 1.1. Any trivial stratified surface is isomorphic to a stratified surface from the list:

(1) a sphere $S^{2}$ without special points $(\mu=-2)$;

(2) a projective plane $\mathbb{R} P^{2}$ without special points $(\mu=-1)$;

(3) a disk $D^{2}$ without special points $(\mu=-1)$;

(4) a sphere $\left(S^{2}, p\right)$ with a single interior special point $p(\mu=-1)$;

(5) a disk $\left(D^{2}, q\right)$ with a single special boundary point and without special interior points $\left(\mu=-\frac{1}{2}\right)$;

(6) a sphere $\left(S^{2}, p_{1}, p_{2}\right)$ with two interior special points $(\mu=0)$;

(7) a projective plane $\left(\mathbb{R} P^{2}, p\right)$ with a single interior special point $(\mu=0)$;

(8) a torus $T^{2}$ without special points $(\mu=0)$;

(9) a Klein bottle $K l$ without special points $(\mu=0)$;

(10) a disk $\left(D^{2}, p\right)$ with a single interior special point and without boundary special points $(\mu=0)$;

(11) a disk $\left(D^{2}, q_{1}, q_{2}\right)$ with two boundary special points and without interior special points $(\mu=0)$;

(12) a Möbius band $M b$ without special points $(\mu=0)$;

(13) a cylinder $C y l$ without special points $(\mu=0)$.

Let $\Omega$ be a stratified surface. A generic not self-intersecting curve $\gamma \subset \Omega$ is called a cut. Generic means that $\gamma$ has no special points and either is a (closed) contour without boundary points of $\Omega$ or it is a segment, whose ends belong to the boundary of $\Omega$ and all interior points are interior points of the surface. The cuts form 9 topological classes described in [3]. A set of pairwise nonintersecting cuts is called a cut system.

Let $\Gamma$ be a cut system of a stratified surface $\Omega$. Consider compactification $\widetilde{\Omega}$ of $\Omega \backslash \Gamma$ by pairs $(x, c)$, where $x \in \gamma \subset \Gamma$ and $c$ is a coorientation of the cut $\gamma$ in a neighbourhood of $x$. Denote by $\Omega / \Gamma$ a surface obtained by contracting each connected component $C_{i}$ of $\widetilde{\Omega} \backslash(\Omega \backslash \Gamma)$ into a point $c_{i}$. We assume that $\Omega_{\#}=\Omega / \gamma$ is a stratified surface. Its special points are the special points of $\Omega$ and the points $c_{i}$.

1.2. In this subsection, following [3], we define a tensor category $\mathcal{C}$ of stratified surfaces with a set $\mathcal{O}$ of local orientations of special points. $A$ set of local orientations means that for any special point $r \in Q$ of stratified surface $\Omega$ we fix an orientation $o_{r}$ of its small neighbourhood. 
A set $\mathcal{O}$ of local orientations is said admissible, iff either $\Omega$ is orientable surface and all local orientations are induced by an orientation of $\Omega$ or $\Omega$ is non-orientable surface and all local orientations at all special points from any boundary contour $\omega_{i}$ are compatible with one of the orientations of $\omega_{i}$. Moreover, we consider that any boundary contour contain at least one special point.

Lemma 1.2 Let $\left(\Omega^{\prime}, \mathcal{O}^{\prime}\right)$ and $\left(\Omega^{\prime \prime}, \mathcal{O}^{\prime \prime}\right)$ be two pairs, consisting of stratified surfaces and admissible sets of local orientations at special points. If stratified surfaces $\Omega^{\prime}$ and $\Omega^{\prime \prime}$ are isomorphic then there exists an isomorphism $\phi: \Omega^{\prime} \rightarrow \Omega^{\prime \prime}$ such that $\phi\left(\mathcal{O}^{\prime}\right)=\mathcal{O}^{\prime \prime}$.

The proof follows from standard properties of surfaces.

Pairs $(\Omega, \mathcal{O})$ of stratified surfaces $\Omega$ with sets of local orientations $\mathcal{O}$ at their special points are objects of the basic category $C$. Morphisms are any combinations of the morphisms of types 1)-4).

1) Isomorphism $\phi:(\Omega, \mathcal{O}) \rightarrow\left(\Omega^{\prime}, \mathcal{O}^{\prime}\right)$. By a definition, $\phi$ is an isomorphism $\phi: \Omega \rightarrow \Omega^{\prime}$ of stratified surfaces compatible with local orientations at special points.

2) Change of local orientations $\psi:(\Omega, \mathcal{O}) \rightarrow\left(\Omega, \mathcal{O}^{\prime}\right)$. Thus, there is one such morphism for any pair $\left(\mathcal{O}, \mathcal{O}^{\prime}\right)$ of sets of local orientations on a stratified surface $\Omega$.

3) Cutting $\eta:(\Omega, \mathcal{O}) \rightarrow\left(\Omega_{\#}, \mathcal{O}_{\#}\right)$. The morphism $\eta$ depends on a cut system $\Gamma$ endowed with orientations of all cuts $\gamma \in \Gamma$. $\Omega_{\#}$ is defined as contracted cut surface $\Omega / \Gamma$. Stratified surface $\Omega_{\#}$ inherits special points of $\Omega$ and local orientations at any of them. The orientations of cuts induce the local orientations at other special points.

Define a 'tensor product' $\theta:\left(\Omega^{\prime}, \mathcal{O}^{\prime}\right) \times\left(\Omega^{\prime \prime}, \mathcal{O}^{\prime \prime}\right) \rightarrow(\Omega, \mathcal{O})$ of two pairs $\left(\Omega^{\prime}, \mathcal{O}^{\prime}\right)$ and $\left(\Omega^{\prime \prime}, \mathcal{O}^{\prime \prime}\right)$ as their disjoint union $(\Omega, \mathcal{O})=\left(\Omega^{\prime} \sqcup \Omega^{\prime \prime}, \mathcal{O}^{\prime} \sqcup \mathcal{O}^{\prime \prime}\right)$.

Subcategories of the basis category $\mathcal{C}$ are called $2 D$ categories. The basic category $\mathcal{C}$ has subcategories $\mathcal{C}_{g, s, \epsilon}$, where $g$ is either an integer or half-integer nonnegative number or $\infty, s$ is an integer nonnegative number or $\infty$ and $\epsilon=0,1$. The objects of $\mathcal{C}_{g, 0,0}$ are all pairs $(\Omega, \mathcal{O})$, where $\Omega$ is a stratified surface of type $(\widetilde{g}, \epsilon, m)$ and $\widetilde{g} \leqslant g$. For $s>0$ objects of $\mathcal{C}_{g, s, 0}$ are all pairs $(\Omega, \mathcal{O})$, where $\Omega$ is a stratified surface of type $\left(\widetilde{g}, \epsilon, m, m_{1}, \ldots, m_{\widetilde{s}}\right), \widetilde{g} \leqslant g$, and $\widetilde{s} \leqslant 2(g-\widetilde{g})+s$. Thus, $\mathcal{C}=\mathcal{C}_{\infty, \infty, 0}$. The category $\mathcal{C}_{g, s, 1}$ is a subcategory of $\mathcal{C}_{g, s, 0}$. Its objects are all objects $(\Omega, \mathcal{O})$ of $\mathcal{C}_{g, s, 0}$ such that $\Omega$ is an orientable surface. Denote by $\mathcal{C}_{g, s, 1,0}$ subcategory of $\mathcal{C}_{g, s, 1}$, consisting of $(\Omega, \mathcal{O})$, where $\mathcal{O}$ is generated by some global orientation of $\Omega$. This global orientation is marked by the same symbol $\mathcal{O}$.

1.3. Below we define a structure functor $(\Omega, \mathcal{O}) \rightarrow V(\Omega, \mathcal{O})$ from the basic category of surfaces to the category of vector spaces [3].

Let $\left\{X_{m} \mid m \in M\right\}$ be a finite set of $n=|M|$ vector spaces $X_{m}$. The action of the symmetric group $S_{n}$ on the set $\{1, \ldots, n\}$ induces 
its action on the linear space $\left(\oplus_{\sigma:\{1, \ldots, n\} \leftrightarrow M} X_{\sigma(1)} \otimes \cdots \otimes X_{\sigma(n)}\right)$, an element $s \in S_{n}$ brings a summand $X_{\sigma(1)} \otimes \cdots \otimes X_{\sigma(n)}$ to the summand $X_{\sigma(s(1))} \otimes \cdots \otimes X_{\sigma(s(n))}$. Denote by $\otimes_{m \in M} X_{m}$ the subspace of all invariants of this action.

The vector space $\otimes_{m \in M} X_{m}$ is canonically isomorphic to a tensor product of all $X_{m}$ in any fixed order, the isomorphism is a projection of the vector space $\otimes_{m \in M} X_{m}$ to the summand that is equal to the tensor product of $X_{m}$ in a given order. Assume that all $X_{m}$ are equal to a fixed vector space $X$. Then any bijection $M \leftrightarrow M^{\prime}$ of sets induces the isomorphism $\otimes_{m \in M} X_{m} \leftrightarrow \otimes_{m^{\prime} \in M^{\prime}} X_{m^{\prime}}$.

Let $A$ and $B$ be finite dimensional vector spaces over a field $\mathbb{K}$ endowed with involutive linear transformations $A \rightarrow A$ and $B \rightarrow B$, which we denote by $x \mapsto x^{*}(x \in A)$ and $y \mapsto y^{*}(y \in B)$ resp.

Let $(\Omega, \mathcal{O})$ be a pair, consisting of a stratified surface $\Omega$ and a set $\mathcal{O}$ of local orientations at its special points. Denote by $\Omega_{a}$ the set of all interior special points and by $\Omega_{b}$ the set of all boundary special points. Put also $\Omega_{0}=\Omega_{a} \sqcup \Omega_{b}$. Assign a copy $A_{p}$ of a vector space $A$ to any point $p \in \Omega_{a}$ and a copy $B_{q}$ of a vector space $B$ to any point $q \in \Omega_{b}$. Put $V(\Omega, \mathcal{O})=V_{\Omega}=\left(\otimes_{p \in \Omega_{a}} A_{p}\right) \otimes\left(\otimes_{q \in \Omega_{b}} B_{q}\right)$.

The group $\Sigma(\Omega)$ of transpositions of $\Omega_{b}$ acts naturel on $V_{\Omega}$.

For any morphism of pairs $(\Omega, \mathcal{O}) \rightarrow\left(\Omega^{\prime}, \mathcal{O}^{\prime}\right)$ define a morphism of vector spaces $V_{\Omega} \rightarrow V_{\Omega^{\prime}}$ as follows:

1) An isomorphism $\phi:(\Omega, \mathcal{O}) \rightarrow\left(\Omega^{\prime}, \mathcal{O}^{\prime}\right)$ induces the isomorphism $\phi_{*}: V_{\Omega} \rightarrow V_{\Omega^{\prime}}$ because $\phi$ generates the bijections $\Omega_{a} \leftrightarrow \Omega_{a}^{\prime}$ and $\Omega_{b} \leftrightarrow \Omega_{b}^{\prime}$ of sets of special points.

2) For a change of local orientations $\psi:(\Omega, \mathcal{O}) \rightarrow\left(\Omega, \mathcal{O}^{\prime}\right)$ define a linear map $\psi_{*}: V_{\Omega} \rightarrow V_{\Omega}$ as $\left(\otimes_{r \in \Omega_{0}} \psi_{r}\right)$, where for any $r \in \Omega_{0}$

$$
\psi_{r}(x)=\left\{\begin{array}{l}
x, \text { if } o_{r}=o_{r}^{\prime} \\
x^{*}, \quad \text { if } o_{r}=-o_{r}^{\prime}
\end{array}\right.
$$

3) In order to define a morphism $\eta_{*}: V_{\Omega} \rightarrow V_{\Omega_{\#}}$ for any cutting morphism $\eta:(\Omega, \mathcal{O}) \rightarrow\left(\Omega_{\#}, \mathcal{O}_{\#}\right)$ we need to fix elements $\widehat{K}_{A, *} \in A \otimes A$, $\widehat{K}_{B, *} \in B \otimes B$ and $U \in A$. (The notation will be clear from the sequel.)

Evidently, it is sufficient to define $\eta_{*}$ for an arbitrary oriented cut $\gamma \subset \Omega$. In this case we have a canonical isomorphism $V_{\Omega_{\#}}=V_{\Omega} \otimes X$, where

$$
X=\left\{\begin{array}{l}
A \otimes A, \text { if } \gamma \text { is a coorientable contour } \\
B \otimes B, \text { if } \gamma \text { is a segment, } \\
A, \text { if } \gamma \text { is a noncoorientable cut. }
\end{array}\right.
$$

For $x \in V_{\Omega}$ put $\eta_{*}(x)=x \otimes z$, where $z$ is either $\widehat{K}_{A, *}$, or $\widehat{K}_{B, *}$, or $U$ resp.

Finally, for a 'tensor product' $\theta:\left(\Omega^{\prime}, \mathcal{O}^{\prime}\right) \times\left(\Omega^{\prime \prime}, \mathcal{O}^{\prime \prime}\right) \rightarrow\left(\Omega^{\prime} \sqcup \Omega^{\prime \prime}, \mathcal{O}^{\prime} \sqcup\right.$ $\left.\mathcal{O}^{\prime \prime}\right)$ there is evident canonical linear map $\theta_{*}: V_{\Omega^{\prime}} \otimes V_{\Omega^{\prime \prime}} \rightarrow V_{\Omega^{\prime} \sqcup \Omega^{\prime \prime}}$. 


\section{Topological Field Theory over a Functor}

2.1. Let $\mathcal{R}$ be a category of triples $(W, \rho, \Sigma)$ where $W$ is a vector spaces over a field $\mathbb{K}, \Sigma$ is a group, and $\rho: \Sigma \rightarrow A u t(W)$ is a homomorphism. Morphisme $(W, \rho, \Sigma) \rightarrow\left(W^{\prime}, \rho^{\prime}, \Sigma^{\prime}\right)$ is a pair of isomorphisms $\left(\vartheta_{W}\right.$ : $\left.W \rightarrow W^{\prime}, \vartheta_{\Sigma}: \Sigma \rightarrow \Sigma^{\prime}\right)$ such that $\vartheta_{W} \rho=\rho^{\prime} \vartheta_{\Sigma} \vartheta_{W}$.

Let $\mathcal{T}$ be a functor from a $2 D$ category to $\mathcal{R}$ such that $\mathcal{T}(\Omega, \mathcal{O})=$ $(W(\Omega, \mathcal{O}), \rho(\Omega, \mathcal{O}), \Sigma(\Omega, \mathcal{O}))$, where $\Sigma(\Omega, \mathcal{O})=\Sigma(\Omega)$ is the group of transpositions of $\Omega_{b}$

We consider that $\mathcal{T}(\Omega, \mathcal{O})=(\mathbb{K}, \rho, \Sigma)$, where $\rho(\Sigma)$ is the identical map, if $\Omega$ is a trivial stratified surface, and $\mathcal{T}(\pi)$ is the identical morphism, if $\pi$ is a morphism of trivial stratified surfaces.

A Topological Field Theory over $\mathcal{T}$ is a set

$$
\mathcal{F}=\left\{A, x \mapsto x^{*}, B, y \mapsto y^{*}, \mathcal{T}, \Phi_{(\Omega, \mathcal{O})}\right\},
$$

where $\left(A, x \mapsto x^{*}\right)$ and $\left(B, y \mapsto y^{*}\right)$ are finite dimensional vector spaces over $\mathbb{K}$ endowed with involute linear transformation and $\left\{\Phi_{(\Omega, \mathcal{O})}\right\}$ is a family of linear operators $\Phi_{(\Omega, \mathcal{O})}: V_{\Omega} \rightarrow W(\Omega, \mathcal{O})$, where $V_{\Omega}$ is the image of $(\Omega, \mathcal{O})$ by the structural functor for $\left\{A, x \mapsto x^{*}, B, y \mapsto y^{*}\right\}$ and $\mathcal{T}(\Omega, \mathcal{O})=(W(\Omega, \mathcal{O}), \rho(\Omega, \mathcal{O}), \Sigma(\Omega, \mathcal{O}))$.

The set $\mathcal{F}$ is called a Topological Field Theory, if the following axioms are satisfied

$0^{\circ}$ Algebraic invariance

For any $\sigma \in \Sigma(\Omega, \mathcal{O})$ it is required that

$$
\Phi_{(\Omega, \mathcal{O})}(\sigma(x))=\rho(\Omega, \mathcal{O})(\sigma)\left(\Phi_{(\Omega, \mathcal{O})}(x)\right) .
$$

\section{$1^{\circ}$ Topological invariance}

For any isomorphism of pairs $\phi:(\Omega, \mathcal{O}) \rightarrow\left(\Omega^{\prime}, \mathcal{O}^{\prime}\right)$ it is required that

$$
\Phi_{\left(\Omega^{\prime}, \mathcal{O}^{\prime}\right)}\left(\phi_{*}(x)\right)=\mathcal{T}(\phi) \Phi_{(\Omega, \mathcal{O})}(x) .
$$

$2^{\circ}$ Invariance of a change of local orientations.

For any change of local orientations $\psi:(\Omega, \mathcal{O}) \rightarrow\left(\Omega, \mathcal{O}^{\prime}\right)$ it is required that

$$
\Phi_{\left(\Omega, \mathcal{O}^{\prime}\right)}\left(\psi_{*}(x)\right)=\mathcal{T}(\psi) \Phi_{(\Omega, \mathcal{O})}(x) .
$$

$3^{\circ}$ Nondegeneracy.

Define first a bilinear form $\left(x, x^{\prime}\right)_{A}$ on the vector space $A$. Namely, let $(\Omega, \mathcal{O})$ be a pair, where $\Omega$ is a sphere with 2 interior special points $p, p^{\prime}$ and the set $\mathcal{O}=\left\{o_{p}, o_{p^{\prime}}\right\}$ is such that local orientations $o_{p}, o_{p^{\prime}}$ induce the same global orientations of the sphere. Put $\left(x, x^{\prime}\right)_{A}=\Phi_{(\Omega, \mathcal{O})}\left(x_{p} \otimes\right.$ $x_{p^{\prime}}^{\prime}$, where $x_{p}$ and $x_{p^{\prime}}^{\prime}$ are images of $x \in A$ and $x^{\prime} \in A$ in $A_{p}$ and $A_{p^{\prime}}$ resp. The correctness of this definition follows from axioms $1^{\circ}$ and $2^{\circ}$. Evidently, $\left(x, x^{\prime}\right)_{A}$ is a symmetric bilinear form.

Similarly, define a bilinear form $\left(y, y^{\prime}\right)_{B}$ on the vector space $B$, using a disc with 2 boundary special points $q, q^{\prime}$ instead of a sphere with two interior special points $p, p^{\prime}$. As in the previous case, local orientations 
$o_{q}, o_{q^{\prime}}$ must induce the same global orientations of the disc. Evidently, $\left(y, y^{\prime}\right)_{B}$ is a symmetric bilinear form.

It is required that forms $\left(x, x^{\prime}\right)_{A}$ and $\left(y, y^{\prime}\right)_{B}$ are nondegenerate. $4^{\circ}$ Cut invariance.

Axioms $1^{\circ}-3^{\circ}$ allows us to choose elements $\widehat{K}_{A, *} \in A \times A, \widehat{K}_{B, *} \in B \times$ $B$ and $U \in A$. Indeed, any nondegenerate bilinear form on the vector space $X$ canonically defines [3], Section 2] the tensor Casimir element $\widehat{K}_{X} \in X \otimes X$. Taking forms $\left(x, x^{\prime}\right)_{A, *}=\left(x, x^{\prime *}\right)_{A}$ and $\left(y, y^{\prime}\right)_{B, *}=$ $\left(y, y^{\prime *}\right)_{B}$, we obtain elements $\widehat{K}_{A, *}$ and $\widehat{K}_{B, *}$.

A linear form $\Phi_{(\Omega, \mathcal{O})}$ for a projective plane $\Omega$ with one interior special point is an element of the vector space dual to $A$. We denote by $U$ the image of this element in $A$ under the isomorphism induced by nondegenerate bilinear form $\left(x, x^{\prime}\right)_{A}$.

We shall use just these elements for morphisms $\eta_{*}$ of type 3 ).

For any cut system $\Gamma$ endowed with orientations of all cuts it is required that

$$
\Phi_{\left(\Omega_{\#}, \mathcal{O}_{\#}\right)}\left(\eta_{*}(x)\right)=\mathcal{T}(\eta) \Phi_{(\Omega, \mathcal{O})}(x) .
$$

$5^{\circ}$ Multiplicativity.

For the product $\theta:\left(\Omega^{\prime}, \mathcal{O}^{\prime}\right) \times\left(\Omega^{\prime \prime}, \mathcal{O}^{\prime \prime}\right) \rightarrow\left(\Omega^{\prime} \sqcup \Omega^{\prime \prime}, \mathcal{O}^{\prime} \sqcup \mathcal{O}^{\prime \prime}\right)$ of any two pairs $\left(\Omega^{\prime}, \mathcal{O}^{\prime}\right)$ and $\left(\Omega^{\prime \prime}, \mathcal{O}^{\prime \prime}\right)$ it is required that

$$
\Phi_{\left(\Omega_{1} \sqcup \Omega_{2}, \mathcal{O}_{1} \sqcup \mathcal{O}_{2}\right)}\left(\theta_{*}\left(x_{1} \otimes x_{2}\right)\right)=\Phi_{\left(\Omega_{1}, \mathcal{O}_{1}\right)}\left(x_{1}\right) \otimes \Phi_{\left(\Omega_{2}, \mathcal{O}_{2}\right)}\left(x_{2}\right) .
$$

2.2. Let us consider now some example of Topological Field Theory on $2 D$ categories. In this subsection we consider only functors $\mathcal{T}(\Omega, \mathcal{O})=$ $(W(\Omega, \mathcal{O}), \rho(\Omega, \mathcal{O}), \Sigma(\Omega, \mathcal{O}))$, such that $\rho(\Omega, \mathcal{O})(\sigma)$ is the identical map for all $\sigma \in \Sigma(\Omega, \mathcal{O})$.

1) Topological Field Theory on $\mathcal{C}_{g, s, \epsilon}$ (over trivial functor).

Consider the functor, corresponding the field $\mathbb{K}$ to all objects $(\Omega, \mathcal{O})$ and corresponding identical map to any morphism.

a) This gives an Atiayh-Witten 2D Topological Field Theory [2, 13] for the category $\mathcal{C}_{\infty, 0,1}$ and involution $x^{*}=x$.

b) The category $\mathcal{C}_{\infty, \infty, 1}$ and the involutions $x^{*}=x, y^{*}=y$ gives 'an Open-Closed' Topological Field Theory in the sense of Lazaroiu [8].

c) The category $\mathcal{C}_{\infty, \infty, \epsilon}$ gives a Klein Topological Field Theory in the sense of [3] (without units) .

2) Cohomological Field Theories on $\mathcal{C}_{g, s, \epsilon}$.

Recall that a Klein surface of type $(g, s, \epsilon)$ is a surface of type $(g, s, \epsilon)$, endowed with a dianalytic structure, i.e., an atlas with holomorphic and antiholomorphic transitions functions [1]. It is equivalent to a real algebraic curve (for an information about real algebraic curves see [11]). A Klein surface of type $G=\left(g, \epsilon, m, m_{1}, \ldots, m_{s}\right)$ is called a Klein surface with special points of type $G$. The moduli space of Klein surfaces is constructed in [10]. Let $H^{*}\left(\bar{M}_{G}, \mathbb{K}\right)$ be a cohomological algebra of Deline-Mumford compactification of the space of Klein surfaces of type 
$G$. An identification of special points gives some embeddings $\bar{M}_{G_{1}} \times$ $\bar{M}_{G_{2}} \rightarrow \bar{M}_{G}$ and $\bar{M}_{G_{1}} \rightarrow \bar{M}_{G}$ by analogy with [9].

Cohomological Field Theory in our conception is defined as the Topological Field Theory over the functor $\mathcal{T}$ that associate the algebra $H^{*}\left(\bar{M}_{G}, \mathbb{K}\right)$ with each object $(\Omega, \mathcal{O})$, where $G$ is the type of $\Omega$, and associate the homomorphisms generated by the embeddings $\bar{M}_{G_{1}} \times \bar{M}_{G_{2}} \rightarrow \bar{M}_{G}$ and $\bar{M}_{G_{1}} \rightarrow \bar{M}_{G}$ with the cutting morphisms. Here $x^{*}=x, y^{*}=y$ and the other morphisms are the same as that for the trivial functor.

This definition takes the (complete) Cohomological Field Theory as regards to [9] for subcategories $\mathcal{C}_{0,0,1}\left(\mathcal{C}_{\infty, 0,1}\right)$.

2.3. According to [9], the Cohomological Field Theory over $\mathcal{C}_{0,0,1}$ generate some deformations of Atiayh-Witten 2D Topological Field Theory in genus 0. These deformations are described by formal Frobenius manifolds, i.e., formal solutions of WDVV equations [5, 14.

Our goal is the construction of a functor on $\mathcal{C}_{0,1,1}^{0}$ such that Topological Field Theory over this functor generate deformations of OpenClosed Topological Field Theories in genus 0. We shall prove that these deformations are described by formal solutions of some noncommutative analogues of WDVV equations.

\section{Stable Field Theories}

3.1. Let $\Omega=(\Omega, \mathcal{O})$ be a oriented stratified surface and $\mathcal{O}$ be generated by some orientation. (Here and later we omit the mark of orientation $\mathcal{O}$, if it is constant in the construction). The orientation of $\Omega$ gives a natural sense to inequalities $b_{1}<b_{2}<b_{3}$ for points from a connected component of the boundary of $\Omega$. For $C \subset \Omega_{b}$ denote by $\Sigma^{\circ}(C)$ the group of transpositions $\sigma \in \Sigma(\Omega)$, such that $\sigma\left(b_{1}\right)<\sigma\left(b_{2}\right)<\sigma\left(b_{3}\right)$ if $b_{1}<b_{2}<b_{3}$ and $b_{1}, b_{2}, b_{3} \in \partial \Omega$.

A pair $(\Gamma, \sigma)$, where $\Gamma \subset \Omega$ is a cut system and $\sigma \in \Sigma(\Omega)$ is called a tiling, if all connected components of $\Omega / \Gamma$ are nontrivial. A tiling $(\Gamma, \sigma)$, where $\Gamma=\emptyset$, is also assumed and it is called empty tiling.

Two tiling $\left(\Gamma^{\prime}, \sigma^{\prime}\right)$ and $\left(\Gamma^{\prime \prime}, \sigma^{\prime \prime}\right)$ are called isomorphic, if there exists a homeomorphism $\psi: \Omega \rightarrow \Omega$, preserving the orientation, moving $\sigma^{\prime}$ to $\sigma^{\prime \prime}$ and such that $\psi\left(\Gamma^{\prime}\right)=\Gamma^{\prime \prime}$. An isomorphic class of a tiling is called a diagram. Let $[\Gamma, \sigma]$ be the diagram corresponding to a tiling $(\Gamma, \sigma)$.

Denote by $\mathbb{K}(\Omega)$ the vector space over $\mathbb{K}$ generated by all diagrams of $\Omega$.

Consider a tiling $(\Gamma, \sigma)$, corresponding to a diagram $T \in \mathbb{K}(\Omega)$. A connected component of $\Omega \backslash \Gamma$ is called a vertex of the tiling. Denote by $V(T)$ the set of vertices of $T$.

Let $\Omega_{v}^{T}$ be the connected component of $\Omega / \Gamma$, corresponding to a vertex $v$. Let $A_{v}^{T}$ and $B_{v}^{T}$ be the set of interior and boundary special points of $\Omega_{v}^{T}$. We can associate $\sigma^{*} \in \Sigma(\Omega)$ to any $\sigma_{v}^{T} \in \Sigma\left(\Omega_{v}^{T}\right)$, 
considering that $\sigma^{*}\left(b_{1}\right)<\sigma^{*}\left(b_{2}\right)<\sigma^{*}\left(b_{3}\right)$ if $b_{1}, b_{2}, b_{3} \notin \Omega_{v}^{T}$ belong to the same connected component of $\Omega \backslash \Gamma$ and $b_{1}<b_{2}<b_{3}$. Moreover, we can consider any cut system on $\Gamma_{v}^{T} \in \Omega_{v}^{T}$ as a cut system on $\Omega$. Thus, a diagram $S=\left(\Gamma_{v}^{T}, \sigma_{v}^{T}\right) \in \mathbb{K}\left(\Omega_{v}^{T}\right)$ generates the diagram $(T, v, S)=\left(\Gamma \cup \Gamma_{v}^{T}, \sigma^{*} \sigma\right) \in(\mathbb{K}(\Omega))$.

3.2. A tiling $(\Gamma, \sigma)$ is called simple, if $\Gamma$ consist of one cut. A simple tiling is called $A$-tiling, if $\Gamma$ is a closed contour homotopic to zero, $B$ tiling, if $\Gamma$ is a segment, dividing the surface, $C$-tiling, if $\Gamma$ is a closed contour nonhomotopic to zero, and D-tiling, if $\Gamma$ is a segment, nondividing the surface. Diagrams, corresponding to $A(B, C, D)$, empty tilings are called $A(B, C, D)$ empty diagram.

Let $C_{1}$ and $C_{2}$ be subsets of a surface $\Omega$. By $r_{A}\left(C_{1} \mid C_{2}\right)$ (respectively, $r_{B}\left(C_{1} \mid C_{2}\right)$ ) denote the set of $A-$ (respectively, $B-$ ) diagrams, corresponding to $(\Gamma, \sigma)$, where $\Gamma$ divide $C_{1}$ and $C_{2}$.

For a sphere $\Omega$ and $a_{1}, a_{2}, a_{3}, a_{4} \in \Omega_{a}$ put $r_{\Omega}\left(a_{1}, a_{2} \mid a_{3}, a_{4}\right)=$ $r_{A}\left(a_{1}, a_{2} \mid a_{3}, a_{4}\right)$.

Let $\Omega$ be a disk, $b_{1}, b_{2}, b_{3}, b_{4} \in \Omega_{b}$ and $b_{1}<b_{2}<b_{3}<b_{4}$. Denote by $r_{\Omega}\left(b_{1}, b_{2} \mid b_{3}, b_{4}\right)$ the set of all diagrams, belonging to $r_{B}\left(\sigma\left(b_{1}\right) \cup\right.$ $\left.\sigma\left(b_{2}\right) \mid \sigma\left(b_{3}\right), \cup \sigma\left(b_{4}\right)\right)$ and corresponding to $(\Gamma, \sigma)$, where $\sigma \in \Sigma^{\circ}\left(\sigma\left(b_{1}\right) \cup\right.$ $\left.\sigma\left(b_{2}\right) \cup \sigma\left(b_{3}\right) \cup \sigma\left(b_{4}\right)\right) \cap \Sigma\left(\Omega_{b}-\left(\sigma\left(b_{1}\right) \cup \sigma\left(b_{2}\right) \cup \sigma\left(b_{3}\right) \cup \sigma\left(b_{4}\right)\right)\right)$.

Let $\Omega$ be a disk $a \in \Omega_{a}, b_{1}, b_{2} \in \Omega_{b}, \sigma \in \Sigma(\Omega)$. Denote by $r_{\Omega}\left(b_{1}, b_{2} \mid a\right)$ the set of all diagrams belonging to $r_{B}\left(\sigma\left(b_{1}\right) \cup \sigma\left(b_{2}\right) \mid a\right)$ and corresponding to $(\Gamma, \sigma)$, where $\sigma \in \Sigma\left(\Omega_{b}-\left(\sigma\left(b_{1}\right) \cup \sigma\left(b_{2}\right)\right)\right)$ and any ends $b$ of $\Gamma$ satisfy $\sigma\left(b_{1}\right)<b<\sigma\left(b_{2}\right)$.

Let $\Omega$ be a disk $a_{1}, a_{2} \in \Omega_{a}, b \in \Omega_{b}$. Put $r_{\Omega}\left(a_{1}, a_{2} \mid b\right)=r_{A}\left(a_{1}, a_{2} \mid b\right)$. Denote by $r_{\Omega}\left(a_{1}, b \mid a_{2}\right) r_{\Omega}\left(a_{2}, b \mid a_{1}\right)$ the diagrams corresponding to $\left(\Gamma_{1} \cup\right.$ $\left.\Gamma_{2}, \sigma\right)$, where $\left(\Gamma_{1}, \sigma\right)$ from $r_{B}\left(a_{1} \cup \sigma(b) \mid a_{2}\right),\left(\Gamma_{2}, \sigma^{\prime \prime}\right)$ from $r_{B}\left(a_{2} \cup \sigma(b) \mid a_{1}\right)$, and $\sigma \in \Sigma\left(\Omega_{b}-b\right)$.

Let $\Omega$ be a cylinder. Denote by $r_{\Omega}^{\sigma}(C)$ and $r_{\Omega}^{\sigma}(D)$ the set of all $C-$ and $D$-diagrams respectively.

Denote by $H^{*}(\Omega)$ the set of linear functions $l: \mathbb{K}(\Omega) \rightarrow \mathbb{K}$, that is equal 0 on all elements in the forms:

1)

$$
[\phi(\Gamma), \sigma(\phi) \sigma]-[\Gamma, \sigma]
$$

for any tiling $(\Gamma, \sigma)$ of $\Omega$, any isomorphism $\phi:(\Omega, \mathcal{O}) \rightarrow(\Omega, \mathcal{O})$ and the transposition $\sigma(\phi)=\left.\phi\right|_{\Omega_{b}}$

2)

$$
\sum_{S \in r_{\Omega_{v}^{T}}}(T, v, S)-\sum_{S \in r_{\Omega_{v}^{T}}\left(a_{2}, a_{2}\left|a_{3}\right| a_{3}, a_{4}, a_{1}\right)}(T, v, S)
$$

for any diagram $T \in \mathbb{K}(\Omega)$, any $v \in V(T)$ such that $\Omega_{v}^{T}$, is a sphere, and any pairwise different points $a_{1}, a_{2}, a_{3}, a_{4} \in\left(\Omega_{v}^{T}\right)_{a}$. 
3)

$$
\sum_{S \in r_{\Omega_{v}^{T}}}\left(b_{1}, b_{2} \mid b_{3}, b_{4}\right) \quad(T, v, S)-\sum_{S \in r_{\Omega_{v}^{T}}\left(b_{2}, b_{3} \mid b_{4}, b_{1}\right)}(T, v, S)
$$

for any diagram $T \in \mathbb{K}(\Omega)$, any $v \in V(T)$ such that $\Omega_{v}^{T}$, is a disk, and any $b_{1}, b_{2}, b_{3}, b_{4} \in\left(\Omega_{v}^{T}\right)_{b}$ such that $b_{1}<b_{2}<b_{3}<b_{4}$.

4)

$$
\sum_{S \in r_{\Omega_{v}^{T}}\left(b_{1}, b_{2} \mid a\right)}(T, v, S)-\sum_{S \in r_{\Omega_{v}^{T}}\left(b_{2}, b_{1} \mid a\right)}(T, v, S)
$$

for any diagram $T \in \mathbb{K}(\Omega)$, any $v \in V(T)$ such that $\Omega_{v}^{T}$, is a disk, any $a \in\left(\Omega_{v}^{T}\right)_{a}$, and any different points $b_{1}, b_{2} \in\left(\Omega_{v}^{T}\right)_{b}$.

5)

$$
\sum_{S \in r_{\Omega_{v}^{T}}\left(a_{1}, a_{2} \mid b\right)}(T, v, S)-\sum_{S \in r_{\Omega_{v}^{T}}\left(a_{1}, b \mid a_{2}\right) r_{\Omega_{v}^{T}}\left(a_{2}, b \mid a_{1}\right)}(T, v, S)
$$

for any diagram $T \in \mathbb{K}(\Omega)$, any $v \in V(T)$ such that $\Omega_{v}^{T}$, is a disk with $n+2$ interior and $m+1$ boundary marked points, $a \in\left(\Omega_{v}^{T}\right)_{a}$, and distinct $b_{1}, b_{2} \in\left(\Omega_{v}^{T}\right)_{b}$.

6)

$$
\sum_{S \in r_{\Omega_{v}^{T}}(C)}(T, v, S)-\sum_{S \in r_{\Omega_{v}^{T}}(D)}(T, v, S)
$$

for any diagram $T \in \mathbb{K}(\Omega)$, and any $v \in V(T)$ such that $\Omega_{v}^{T}$, is a cylinder with $n$ interior marked points, with $m+2$ boundary marked points and also any boundary contour contained marked points.

Define the action $\rho(\Omega): \Sigma(\Omega) \rightarrow \operatorname{Aut}\left(H^{*}(\Omega)\right)$ by $\sigma(l)\left(\Gamma, \sigma^{\prime}\right)=$ $l\left(\Gamma, \sigma^{\prime} \sigma\right)$.

Extend our definitions of $\mathbb{K}(\Omega), H^{*}(\Omega)$ and $\rho(\Omega)$ on disconnected sums $\Omega=\Omega_{1} \amalg \Omega_{2}$ by $\mathbb{K}(\Omega)=\mathbb{K}\left(\Omega_{1}\right) \otimes \mathbb{K}\left(\Omega_{2}\right), H^{*}(\Omega)=H\left(\Omega_{1}\right)^{*} \otimes$ $H\left(\Omega_{2}\right)^{*}$. The actions $\rho\left(\Omega_{1}\right)$ and $\rho\left(\Omega_{2}\right)$ generate the action $\rho\left(\Omega_{1}\right)$. This gives a possible to define $H^{*}(\Omega)$ and $\rho(\Omega)$ for any disconnected sums of oriented sphere and disks.

Let $\gamma$ be a simple cut on $\Omega$ and $\Omega_{\#}=\Omega / \gamma$. Denote by $\mathbb{K}_{\gamma}(\Omega) \subset \mathbb{K}(\Omega)$ the subspace generated by all diagrams containing the cut $\gamma$. A diagram $T \in \mathbb{K}\left(\Omega_{\#}\right)$ generates a diagram $\widetilde{T} \in \mathbb{K}_{\gamma}(\Omega)$. This correspondence defines a monomorphism $\varepsilon_{\gamma}: \mathbb{K}\left(\Omega_{\#}\right) \rightarrow \mathbb{K}_{\gamma}(\Omega) \subset K(\Omega)$ and thus a homomorphism $\varepsilon_{\gamma}^{*}: H^{*}(\Omega) \rightarrow H^{*}\left(\Omega_{\#}\right)$.

3.3. Now we introduce a stabilising functor $\mathcal{F}$ on the category $\mathcal{C}_{0,1,1}^{0}$ with structure functor, generated by arbitrary data $\left\{A, x \mapsto x^{*}, B, y \mapsto\right.$ $\left.y^{*}\right\}$.

For local orientations $\mathcal{O}$, generated by a global orientation we put $\mathcal{F}(\Omega, \mathcal{O})=\left(H^{*}(\Omega), \rho(\Omega), \Sigma(\Omega)\right)$. 
For isomorphism $\phi:(\Omega, \mathcal{O}) \rightarrow\left(\Omega^{\prime}, \mathcal{O}^{\prime}\right)$ denote by $\mathcal{F}(\phi): H^{*}(\Omega, \mathcal{O}) \rightarrow$ $H^{*}\left(\Omega^{\prime}, \mathcal{O}^{\prime}\right)$, the isomorphism that it generates.

Chang of global orintation $\psi:(\Omega, \mathcal{O}) \rightarrow\left(\Omega, \mathcal{O}^{\prime}\right)$ is generated by an isomorphism $\phi:(\Omega, \mathcal{O}) \rightarrow\left(\Omega^{\prime}, \mathcal{O}^{\prime}\right)$. Put $\mathcal{F}(\psi)=\mathcal{F}(\phi)$.

For cutting morphism $\eta:(\Omega, \mathcal{O}) \rightarrow\left(\Omega_{\#}, \mathcal{O}_{\#}\right)$ by a cut $\gamma \subset \Omega$ we consider that $\mathcal{F}(\eta)$ is generated by $\varepsilon_{\gamma}^{*}$.

By definition, Topological Field Theory

$$
\mathcal{N}=\left\{A, x \mapsto x^{*}, B, y \mapsto y^{*}, \mathcal{F}, \Phi_{(\Omega, \mathcal{O})}\right\}
$$

with values in a stabilizing functor $\mathcal{F}$ is called a Stable Field Theory.

Remark. It follows from 9] that the codiagram space $H^{*}(\Omega)$ of a sphere with $n$ special points coincides with the cohomological algebra $H^{*}\left(\bar{M}_{0, n}, \mathbb{K}\right)$ of the Deligne-Mumford compactification of moduli space of spheres with $n$ punctures. Thus, for $x^{*}=x$ Stable Field Theory on $2 D$ category $\mathcal{C}_{0,0,1}$ coincides with the Kontsevich-Manin Cohomological Field Theory [7].

\section{System OF DiSK CORRELATION FUnCTIONS}

4.1. Let $A$ and $B$ be vector spaces with basises $\left\{\alpha_{1}, \ldots, \alpha_{n}\right\} \subset$ $A,\left\{\beta_{1}, \ldots, \beta_{m}\right\} \subset B$ and involutions $x \mapsto x^{*}, y \mapsto y^{*}\left(x, x^{*} \in A, y, y^{*} \in\right.$ $B)$. Consider a collection of tensors $\left\{f_{r, \ell}: A^{\otimes r} \otimes B^{\otimes \ell} \rightarrow \mathbb{K}\right\}$. By $<x_{1}, \ldots, x_{k}, y_{1}, \ldots, y_{\ell}>$ denote $f_{k, \ell}\left(x_{1} \otimes \cdots \otimes x_{k} \otimes y_{1} \otimes \cdots \otimes y_{\ell}\right)$, where $x_{i} \in A, y_{i} \in B$. We say that this collection is a System of Disk Correlation Function, if the following conditions hold.

Axiom $1^{0}$ : The value $\left\langle x_{1}, \ldots, x_{k}, y_{1}, \ldots, y_{\ell}\right\rangle$ is invariant under any permutation of $x_{i}$ and cyclic permutation of $y_{i}$.

Axiom $2^{0}:<x_{1}, \ldots, x_{k}, y_{1}, \ldots, y_{\ell}>=<x_{1}^{*}, \ldots, x_{k}^{*}, y_{\ell}^{*}, y_{\ell-1}^{*}, \ldots, y_{1}^{*}>$.

Axiom $3^{0}$ : The bilinear forms $<x_{i}, x_{j}>$ and $<y_{i}, y_{j}>$ are nondegenerate. Denote by $F^{\alpha_{i} \cdot \alpha_{j}}$ and $F^{\beta_{i} \cdot \beta_{j}}$ the inverse matrices for $F_{\alpha_{i} \alpha_{j}}=<\alpha_{i}, \alpha_{j}>$ and $F_{\beta_{i} \beta_{j}}=<\beta_{i}, \beta_{j}>$.

Axiom $4^{0}$ : Let $x_{1}, x_{2}, x_{3}, x_{4}, x^{i} \in A$ and $\mathcal{A}=\left\{x^{1}, \ldots, x^{r}\right\}$. Denote by $<\mathcal{A}\left|x_{1}, x_{2}\right| x_{3}, x_{4}>$ the sum of all numbers as $<$ $x_{1}, x_{2}, x^{\sigma(1)}, \ldots, x^{\sigma(s)}, \alpha_{i}>F^{\alpha_{i} \alpha_{j}}<\alpha_{j}, x_{3}, x_{4}, x^{\sigma(s+1)}, \ldots, x^{\sigma(r)}>$, where $0 \leqslant s \leqslant r, 1 \leqslant i, j \leqslant n$ and $\sigma$ passes through all permutations of $r$ indices.

The axiom says that

$$
<\mathcal{A}\left|x_{1}, x_{2}\right| x_{3}, x_{4}>=<\mathcal{A}\left|x_{4}, x_{1}\right| x_{2}, x_{3}>
$$

for any $\mathcal{A}$ and $x_{1}, x_{2}, x_{3}, x_{4}$.

Axiom $5^{0}: \quad$ Let $x^{i} \in A, \mathcal{A}=\left\{x^{1}, \ldots, x^{r}\right\}$, $y_{1}, y_{2}, y_{3}, y_{4}, y^{i} \in B$, and $\mathcal{B}=\left\{y^{1}, \ldots, y^{p}\right\}$. Denote by $<\mathcal{A}, \mathcal{B}\left|y_{1}, y_{2}\right| y_{3}, y_{4}>$ the sum of all numbers as $<$ $x^{\sigma(1)}, \ldots, x^{\sigma(s)}, y^{\xi(1)}, \ldots, y^{\xi\left(p_{1}\right)}, y_{1}, y^{\xi\left(p_{1}+1\right)}, \ldots, y^{\xi\left(p_{2}\right)}, y_{2}, y^{\xi\left(p_{2}+1\right)}, \ldots, y^{\xi\left(p_{3}\right)}, \beta_{i}>$ $F^{\beta_{i} \beta_{j}}<\beta_{j}, x^{\sigma(s+1)}, \ldots, x^{\sigma(r)}, y^{\xi\left(p_{3}+1\right)}, \ldots, y^{\xi\left(p_{4}\right)}, y_{3}, y^{\xi\left(p_{4}+1\right)}, \ldots, y^{\xi\left(p_{5}\right)}, y_{4}$, $y^{\xi\left(p_{5}+1\right)}, \ldots, y^{\xi(p)}>$, where $0 \leqslant s \leqslant r, 0 \leqslant p_{1} \leqslant p_{2} \leqslant p_{3} \leqslant p_{4} \leqslant p_{5} \leqslant p$, 
$1 \leqslant i, j \leqslant m, \sigma$ passes through all permutations of $r$ indices and $\xi$ passes through all permutations of $p$ indices.

The axiom says that

$$
<\mathcal{A}, \mathcal{B}\left|y_{1}, y_{2}\right| y_{3}, y_{4}>=<\mathcal{A}, \mathcal{B}\left|y_{4}, y_{1}\right| y_{2}, y_{3}>
$$

for any $\mathcal{A}, \mathcal{B}$, and $y_{1}, y_{2}, y_{3}, y_{4}$.

Axiom $6^{0}$ : Let $x, x^{i} \in A, \mathcal{A}=\left\{x^{1}, \ldots, x^{r}\right\}, y_{1}, y_{2}, y^{i} \in B$, and $\mathcal{B}=\left\{y^{1}, \ldots, y^{p}\right\}$ Denote by $<\mathcal{A}, \mathcal{B}|x| y_{1}, y_{2}>$ the sum of all numbers as $<x, x^{\sigma(1)}, \ldots, x^{\sigma(s)}, y^{\xi(1)}, \ldots, y^{\xi\left(p_{1}\right)}, \beta_{i}>F^{\beta_{i} \beta_{j}}<$ $\beta_{j}, x^{\sigma(s+1)}, \ldots, x^{\sigma(r)}, y^{\xi\left(p_{1}+1\right)}, \ldots, y^{\xi\left(p_{2}\right)}, y_{1}, y^{\xi\left(p_{2}+1\right)}, \ldots, y^{\xi\left(p_{3}\right)}, y_{2}, y^{\xi\left(p_{3}+1\right)}, \ldots$, $y^{\xi(p)}>$, where $0 \leqslant s \leqslant r, 0 \leqslant p_{1} \leqslant p_{2} \leqslant p_{3} \leqslant p, 1 \leqslant i, j \leqslant m, \sigma$ passes through all permutations of $r$ indices and $\xi$ passes through all permutations of $p$ indices.

The axiom says that

$$
<\mathcal{A}, \mathcal{B}|x| y_{1}, y_{2}>=<\mathcal{A}, \mathcal{B}|x| y_{2}, y_{1}>
$$

for any $\mathcal{A}, \mathcal{B}, x$, and $y_{1}, y_{2}$.

Axiom $7^{0}$ : Let $x_{1}, x_{2}, x^{i} \in A, \mathcal{A}=\left\{x^{1}, \ldots, x^{r}\right\}, y, y^{i} \in$ $B$, and $\mathcal{B}=\left\{y^{1}, \ldots, y^{p}\right\}$. Denote by $<\mathcal{A}, \mathcal{B}\left|x_{1}, x_{2}\right| y>$ the sum of all numbers as $<x_{1}, x_{2}, x^{\sigma(1)}, \ldots, x^{\sigma(s)}, \alpha_{i}>F^{\alpha_{i} \alpha_{j}}<$ $\alpha_{j}, x^{\sigma(s+1)}, \ldots, x^{\sigma(r)}, y, y^{\xi(1)}, \ldots, y^{\xi(p)}>$, where $0 \leqslant s \leqslant r 1 \leqslant i, j \leqslant n, \sigma$ passes through all permutations of $r$ indices and $\xi$ passes through all permutations of $p$ indices.

Denote by $<\mathcal{A}, \mathcal{B}\left|x_{1}, y\right| x_{2}><\mathcal{A}, \mathcal{B}\left|x_{2}, y\right| x_{1}>$ the sum of all numbers as $<x, x^{\sigma(1)}, \ldots, x^{\sigma(s)}, y, y^{\xi(1)}, \ldots, y^{\xi\left(p_{1}\right)}$, $\beta_{i}^{1}, y^{\xi\left(p_{2}+1\right)}, \ldots, y^{\xi\left(p_{3}\right)}, \quad \quad \beta_{i}^{2}, y^{\xi\left(p_{4}+1\right)}, \ldots, y^{\xi(p)}>$ $F^{\beta_{i}^{1} \beta_{j}^{1}} F^{\beta_{i}^{2} \beta_{j}^{2}} \quad<\quad \beta_{j}^{1}, x^{\sigma(s+1)}, \ldots, x^{\sigma(t)}, y^{\xi\left(p_{1}+1\right)}, \ldots, y^{\xi\left(p_{2}\right)} \quad><$ $\beta_{j}^{2}, x^{\sigma(t+1)}, \ldots, x^{\sigma(r)}, y^{\xi\left(p_{3}+1\right)}, \ldots, y^{\xi\left(p_{4}\right)}>$, where $0 \leqslant s \leqslant r$, $0 \leqslant p_{1} \leqslant p_{2} \leqslant p_{3} \leqslant p_{4} \leqslant p, 1 \leqslant i^{1}, j^{1}, i^{2}, j^{2} \leqslant m, \sigma$ passes through all permutations of $r$ indices and $\xi$ passes through all permutations of $p$ indices.

The axiom says that

$$
<\mathcal{A}, \mathcal{B}\left|x_{1}, x_{2}\right| y>=<\mathcal{A}, \mathcal{B}\left|x_{1}, y\right| x_{2}><\mathcal{A}, \mathcal{B}\left|x_{2}, y\right| x_{1}>
$$

for any $\mathcal{A}, \mathcal{B}, x_{1}, x_{2}$, and $y$.

We say that a system of disk correlation is extended if the following condition holds.

Axiom $8^{0}:$ Let $x^{i} \in A, \mathcal{A}=\left\{x^{1}, \ldots, x^{r}\right\}, y_{1}, y_{2}^{i} \in B$, and $\mathcal{B}=\left\{y^{1}, \ldots, y^{p}\right\}$. Denote by $<\mathcal{A}, \mathcal{B} \mid y_{1}, y_{2} \quad>_{a}$ the sum of all numbers as $<x^{\sigma(1)}, \ldots, x^{\sigma(s)}, y_{1}, y^{\xi(1)}, \ldots, y^{\xi(q)}, \alpha_{i}>F^{\alpha_{i} \alpha_{j}}<$ $\alpha_{j}, x^{\sigma(s+1)}, \ldots, x^{\sigma(r)}, y_{2}, y^{\xi(q+1)}, \ldots, y^{\xi(p)}>$, where $0 \leqslant s \leqslant r, 0 \leqslant q \leqslant p$, $1 \leqslant i, j \leqslant n, \sigma$ passes through all permutations of $r$ indices and $\xi$ passes through all permutations of $p$ indices.

Denote by $<\mathcal{A}, \mathcal{B} \mid y_{1}, y_{2} \quad>_{b}$ the sum of all numbers as $<x^{\sigma(1)}, \ldots, x^{\sigma(r)}, y_{1}, y^{\xi(1)}, \ldots, y^{\xi\left(q_{1}\right)}, \beta_{i}, y^{\xi\left(q_{1}+1\right)}, \ldots, y^{\xi\left(q_{2}\right)}$, 
$y_{2}, y^{\xi\left(q_{2}+1\right)}, \ldots, y^{\xi\left(q_{3}\right)}, \beta_{j}, y^{\xi\left(q_{3}+1\right)}, \ldots, y^{\xi(p)}>F^{\beta_{i} \beta_{j}}$, where $0 \leqslant q_{1} \leqslant$ $q_{2} \leqslant q_{3} \leqslant p, 1 \leqslant i, j \leqslant n, \sigma$ passes through all permutations of $r$ indices and $\xi$ passes through all permutations of $p$ indices.

The axiom says that

$$
<\mathcal{A}, \mathcal{B}\left|y_{1}, y_{2}\right| y>_{a}=<\mathcal{A}, \mathcal{B} \mid y_{1}, y_{2}>_{b}
$$

for any $\mathcal{A}, \mathcal{B}, y_{1}$ and $y_{2}$.

It is easy to prove that the axioms are fulfilled for any bases $\left\{\alpha_{1}, \ldots, \alpha_{n}\right\} \subset A,\left\{\beta_{1}, \ldots, \beta_{m}\right\} \subset B$, if they are fulfilled for one pair of such bases.

4.2. Let $\mathcal{N}=\left\{A, x \mapsto x^{*}, B, y \mapsto y^{*}, \mathcal{F}, \Phi_{(\Omega, \mathcal{O})}\right\}$ be a Stable Field Theory on the category $\mathcal{C}_{0,1,1}^{0}$. Consider the collection of tensors $<$ $x_{1}, \ldots, x_{k}, y_{1}, \ldots, y_{\ell}>_{\mathcal{N}}=\Phi_{(\Omega, \mathcal{O})}(z)(\emptyset, 1)$. Here $\Omega$ is a sphere (if $\ell=0$ ) or a disk (if $\ell>0$ ) with $k$ special interior points $a_{1}, \ldots, a_{k}$ and with $\ell$ special boundaries points $b_{1}, \ldots, b_{\ell}, \emptyset$ is the empty diagram of $\Omega$ and 1 is identical permutation. We assume that the points $b_{1}, \ldots, b_{\ell}$ are ordered by the orientation of disk $\Omega$ and $z=x_{1} \otimes \cdots \otimes x_{k} \otimes y_{1} \otimes \cdots \otimes y_{\ell}$, where $x_{i}$ corresponds to $a_{i}$ and $y_{i}$ corresponds to $b_{i}$. We say that the tensors $\left.<x_{1} \cdots y_{\ell}\right\rangle_{\mathcal{N}}$ are generated by $\mathcal{N}$.

Theorem 4.1. 1) The correspondence $\mathcal{N} \mapsto\left\{\left\langle x_{1}, \ldots y_{\ell}\right\rangle_{\mathcal{N}}\right\}$ is one-to-one correspondence between Stable Field Theories on $\mathcal{C}_{0,1,1}$ and Systems of Disk Correlation Functions. If $\mathcal{N}$ is Stable Field Theories on $\mathcal{C}_{0,2,1}$ then $\left\{<x_{1}, \ldots, y_{\text {ell }}\right\rangle_{\mathcal{N}}$ is an extended systems of disk correlation functions.

Proof: 1) Prove that $\left\{\left\langle x_{1}, \ldots, y_{\ell}\right\rangle_{\mathcal{N}}\right\}$ is a Extended System of Disk Correlation Functions. Axioms $1^{0}, 2^{0}, 3^{0}$ for Systems of Disk Correlation Functions follow from axioms $1^{\circ}, 2^{\circ}, 3^{\circ}$ for Topological Field Theories. The axioms $4^{\circ}-7^{\circ}$ are fulfilled because $\Phi_{(\Omega, \mathcal{O})}(z) \in H^{*}(\Omega)$.

2) Now let $\left\{\left\langle x_{1}, \ldots, y_{\ell}\right\rangle\right\}$ be a System of Disk Correlation Functions. Consider a sphere (if $\ell=0$ ) or a disk (if $\ell>0$ ) $\Omega$ with $k$ special interior points $a_{1}, \ldots, a_{k}$ and with $\ell$ special boundaries points $b_{1}, \ldots, b_{\ell}$. Put $\Phi_{(\Omega, \mathcal{O})}(z)(\emptyset, 1)=<x_{1}, \ldots, x_{k}, y_{1}, \ldots, y_{\ell}>_{\mathcal{N}}$. We assume that the points $b_{1}, \ldots, b_{\ell}$ are ordered by the orientation of disk $\Omega$ and $z=x_{1} \otimes \cdots \otimes x_{k} \otimes y_{1} \otimes \cdots \otimes y_{\ell}$, where $x_{i}$ corresponds to $a_{i}$ and $y_{i}$ corresponds to $b_{i}$. $\emptyset$ is the empty diagram of $\Omega$ and 1 is identical permutation.

Using axioms $0^{\circ}, 4^{\circ}, 5^{\circ}$ for Topological Field Theories we can uniquely continue $\Phi_{(\Omega, \mathcal{O})}(z)$ up to a linear function $l: \mathbb{K}(\Omega, \mathcal{O}) \rightarrow \mathbb{K}$. It is follows from the axioms $4^{0}, 5^{0}, 6^{0}, 7^{0}$ for Systems of Disk Correlation Functions that $l \in H^{*}(\Omega, \mathcal{O})$. It is follows from axioms $1^{0}, 2^{0}, 3^{0}$ for Systems of Disk Correlation Functions that $1^{\circ}, 2^{\circ}, 3^{\circ}$ for Topological Field Theories are fulfilled. 


\section{Structure Equations}

5.1. Let, as above, $A, x \mapsto x^{*}$ and $B, y \mapsto y^{*}$ be vector spaces with involutions. These involutions generate involutions $*$ on $A^{\otimes k} \otimes B^{\otimes \ell}$ by the rule $*\left(x_{1} \otimes \cdots \otimes x_{k} \otimes y_{1} \otimes \cdots \otimes y_{\ell}\right)=\left(x_{1}^{*} \otimes \cdots \otimes x_{k}^{*} \otimes y_{\ell}^{*} \otimes \cdots \otimes y_{1}^{*}\right)$. Fix bases $\left\{\alpha_{1}, \ldots, \alpha_{n}\right\} \subset A$ and $\left\{\beta_{1}, \ldots, \beta_{m}\right\} \subset B$. By definition, we consider that a multiplication of monomials $\alpha_{i_{1}} \otimes \cdots \otimes \alpha_{i_{k}} \otimes \beta_{j_{1}} \otimes \cdots \otimes \beta_{j_{\ell}}$ and $\alpha_{\tilde{i}_{1}} \otimes \cdots \otimes \alpha_{\tilde{i}_{\tilde{k}}} \otimes \beta_{\tilde{j}_{1}} \otimes \cdots \otimes \beta_{\tilde{j}_{\tilde{\ell}}}$ is the monomial $\alpha_{i_{1}} \otimes \cdots \otimes \alpha_{i_{k}} \otimes \alpha_{\tilde{i}_{1}} \otimes$ $\cdots \otimes \alpha_{\tilde{i}_{\tilde{k}}} \otimes \beta_{j_{1}} \otimes \cdots \otimes \beta_{j_{\ell}} \otimes \beta_{\tilde{j}_{1}} \otimes \cdots \otimes \beta_{\tilde{j}_{\tilde{\ell}}}$.

Now consider a tensor series, i.e., a formal series of tensor monomials $F=\sum c\left(i_{1} \cdots i_{k} \mid j_{1} \cdots j_{\ell}\right) \alpha_{i_{1}} \otimes \cdots \otimes \alpha_{i_{k}} \otimes \beta_{j_{1}} \otimes \cdots \otimes \beta_{j_{\ell}}$, where $c\left(i_{1}, \ldots, i_{k} \mid j_{1}, \ldots, j_{\ell}\right) \in \mathbb{K}$. The involution $*$ natural acts on the set of such series. The multiplication of monomial defines the multiplication of formal series.

Define "partial derivative" of tensor series. It is a linear continuation of partial derivative of monomials.

Let $\partial\left(\alpha_{i_{1}} \otimes \cdots \otimes \alpha_{i_{k}} \otimes \beta_{j_{1}} \otimes \cdots \otimes \beta_{j_{\ell}}\right) / \partial \alpha_{i}$ be the sum of monomials as $\alpha_{i_{1}} \otimes \cdots \otimes \alpha_{i_{p-1}} \otimes \alpha_{i_{p+1}} \otimes \cdots \otimes \alpha_{i_{k}} \otimes \beta_{j_{1}} \otimes \cdots \otimes \beta_{j_{\ell}}$ such that $i_{p}=i$.

Similarly, let $\partial\left(\alpha_{i_{1}} \otimes \cdots \otimes \alpha_{i_{k}} \otimes \beta_{j_{1}} \otimes \cdots \otimes \beta_{j_{\ell}}\right) / \partial \beta_{i}$ be the sum of monomials as $\alpha_{i_{1}} \otimes \cdots \otimes \alpha_{i_{k}} \otimes \beta_{j_{1}} \otimes \cdots \otimes \beta_{j_{p-1}} \otimes \beta_{j_{p+1}} \otimes \cdots \otimes \beta_{j_{\ell}}$ such that $j_{p}=i$.

Put $\partial^{2} / \partial \alpha_{i} \partial \alpha_{j}=\left(\partial / \partial \alpha_{i}\right)\left(\partial / \partial \alpha_{j}\right), \quad \partial^{2} / \partial \alpha_{i} \partial \beta_{j} \quad=$ $\left(\partial / \partial \alpha_{i}\right)(\partial / \partial \beta), \quad \partial^{2} / \partial \beta_{i} \partial \beta_{j}=\left(\partial / \partial \beta_{i}\right)\left(\partial / \partial \beta_{j}\right), \partial^{3} / \partial \alpha_{i} \partial \alpha_{j} \partial \alpha_{r}=$ $\left(\partial / \partial \alpha_{i}\right)\left(\partial / \partial \alpha_{j}\right)\left(\partial / \partial \alpha_{r}\right)$

A definition of $\partial^{3}\left(\alpha_{i_{1}} \otimes \cdots \otimes \alpha_{i_{k}} \otimes \beta_{j_{1}} \otimes \cdots \otimes \beta_{j_{\ell}}\right) / \partial \beta_{i} \partial \beta_{j} \partial \beta_{r}$ is more complicated. It ia the sum of monomials as $\alpha_{i_{1}} \otimes \cdots \otimes \alpha_{i_{k}} \otimes$ $\beta_{s_{2}} \otimes \cdots \otimes \beta_{s_{p-1}} \otimes \beta_{s_{p+1}} \otimes \cdots \otimes \beta_{s_{q-1}} \otimes \beta s_{q+1} \otimes \beta_{s_{\ell}}$ such that sequences $\beta_{i}, \beta_{s_{2}}, \ldots, \beta_{s_{p-1}}, \beta_{j}, \beta_{s_{p+1}}, \ldots, \beta_{s_{q-1}}, \beta_{r}, \beta s_{q+1}, \beta_{s_{\ell}}$ and $\beta_{j_{1}}, \ldots, \beta_{j_{\ell}}$ coincide other cyclic permutation.

Monomials $\alpha_{i_{1}} \otimes \cdots \otimes \alpha_{i_{k}} \otimes \beta_{j_{1}} \otimes \cdots \otimes \beta_{j_{\ell}}$ and $\alpha_{\tilde{i}_{1}} \otimes \cdots \otimes \alpha_{\tilde{i}_{k}} \otimes \beta_{\tilde{j}_{1}} \otimes$ $\cdots \otimes \beta_{\tilde{j}_{\ell}}$ are called equivalent, if $\cup_{r=1}^{k} i_{r}=\cup_{r=1}^{k} \widetilde{i}_{k}$ and $\cup_{r=1}^{l} j_{r}=\cup_{r=1}^{l} \widetilde{j}_{r}$. By $\left[\alpha_{i_{1}} \otimes \cdots \otimes \beta_{j_{\ell}}\right]$ denote the equivalence class of $\alpha_{i_{1}} \otimes \cdots \otimes \beta_{j_{\ell}}$. A tensor series $F=\sum c\left(i_{1}, \ldots, i_{k} \mid j_{1}, \ldots, j_{\ell}\right) a_{i_{1}} \otimes \cdots \otimes b_{j_{\ell}}$ generates a series $[F]=\sum c\left[i_{1}, \ldots, i_{k} \mid j_{1}, \ldots, j_{\ell}\right]\left[a_{i_{1}} \otimes \cdots \otimes b_{j_{\ell}}\right]$, where the sum is taken over equivalence classes of monomials and $c\left[i_{1}, \ldots, i_{k} \mid j_{1}, \ldots, j_{\ell}\right]$ is the sum of all coefficients $c\left(\widetilde{i}_{1}, \ldots, \widetilde{j}_{\ell}\right)$, corresponding to monomials from the equivalence class $\left[a_{i_{1}} \otimes \cdots \otimes b_{j_{\ell}}\right]$.

We say that a tensor series $F=\sum c\left(i_{1} \cdots i_{k} \mid j_{1} \cdots j_{\ell}\right) \alpha_{i_{1}} \otimes \cdots \otimes \alpha_{k} \otimes$ $\beta_{j_{1}} \otimes \cdots \otimes \beta_{j_{\ell}}$ is a Structure Series on a space $H=A \oplus B$ with an involution $*$ and bases $\left\{\alpha_{1}, \ldots, \alpha_{n} \mid \beta_{1}, \ldots, \beta_{m}\right\}$, if the following conditions hold

Axiom $1^{0}$. The coefficients $c\left(i_{1} \cdots i_{k} \mid j_{1} \cdots j_{\ell}\right)$ are invariant under all permutations of $\left\{i_{r}\right\}$ and cyclic permutations of $\left\{j_{r}\right\}$.

Axiom $2^{0} . F^{*}=F$. 
Axiom $3^{0}$. The coefficients $c(i, j \mid)$ and $c(\mid i, j)$ generate nondegenerate matrices. By $F_{a}^{\alpha_{i} \alpha_{j}}$ and $F_{b}^{\beta_{i} \beta_{j}}$ denote the inverse matrices of $c(i, j \mid)$ and $c(\mid i, j)$ respectively.

Axiom $4^{0}$.

$$
\begin{aligned}
& {\left[\sum_{p, q=1}^{n} \frac{\partial^{3} F_{a}}{\partial \alpha_{i} \partial \alpha_{j} \partial \alpha_{p}} \otimes F_{a}^{\alpha_{p} \alpha_{q}} \frac{\partial^{3} F_{a}}{\partial \alpha_{q} \partial \alpha_{k} \partial \alpha_{\ell}}\right]=} \\
& {\left[\sum_{p, q=1}^{n} \frac{\partial^{3} F_{a}}{\partial \alpha_{k} \partial \alpha_{j} \partial \alpha_{p}} \otimes F_{a}^{\alpha_{p} \alpha_{q}} \frac{\partial^{3} F_{a}}{\partial \alpha_{q} \partial \alpha_{i} \partial \alpha_{\ell}}\right]}
\end{aligned}
$$

where $F_{a}$ is the part of the tensor series $F$, consisting from all monomials without monomials, containing some $\beta_{i}$.

Axiom $5^{0}$.

$$
\begin{gathered}
{\left[\sum_{p, q=1}^{m} \frac{\partial^{3} F}{\partial \beta_{i} \partial \beta_{j} \partial \beta_{p}} \otimes F_{b}^{\beta_{p} \beta_{q}} \frac{\partial^{3} F}{\partial \beta_{q} \partial \beta_{k} \partial \beta \ell}=\right.} \\
\left.\sum_{p, q=1}^{m} \frac{\partial^{3} F}{\partial \beta_{\ell} \partial \beta_{i} \partial \beta_{p}} \otimes F_{b}^{\beta_{p} \beta_{q}} \frac{\partial^{3} F}{\partial \beta_{q} \partial \beta_{j} \partial \beta_{k}}\right] .
\end{gathered}
$$

Axiom $6^{0}$.

$$
\left[\sum \frac{\partial^{2} F}{\partial \alpha_{i} \partial \beta_{p}} \otimes F_{b}^{\beta_{p} \beta_{q}} \frac{\partial^{3} F}{\partial \beta_{q} \partial \beta_{i} \partial \beta_{j}}\right]=\left[\sum \frac{\partial^{2} F}{\partial \alpha_{i} \partial \beta_{p}} \otimes F_{b}^{\beta_{p} \beta_{q}} \frac{\partial^{3} F}{\partial \beta_{q} \partial \beta_{j} \partial \beta_{i}}\right] .
$$

Axiom $7^{0}$.

$$
\begin{gathered}
{\left[\sum \frac{\partial^{2} F}{\partial \beta_{k} \partial \alpha_{p}} \otimes F_{a}^{\alpha_{p} \alpha_{q}} \frac{\partial^{3} F}{\partial \alpha_{q} \partial \alpha_{i} \partial \alpha_{j}}\right]=} \\
{\left[\sum \frac{\partial^{2} F}{\partial \alpha_{i} \partial \beta_{p}} \otimes F_{b}^{\beta_{p} \beta_{q}} \frac{\partial^{3} F}{\partial \beta_{q} \partial \beta_{k} \partial \beta_{r}} \otimes F_{b}^{\beta_{r} \beta_{\ell}} \frac{\partial^{2} F}{\partial \beta_{\ell} \partial \alpha_{j}}\right] .}
\end{gathered}
$$

We say that the conditions from axioms $1^{0}-7^{0}$ are Structure Equations. They are noncommutative analogues of associativity equations [5, 14].

5.2. Any noncommutative tensor series $F=$ $\sum c\left(i_{1}, \ldots, i_{k} \mid j_{1}, \ldots, j_{\ell}\right) \alpha_{i_{1}} \otimes \cdots \otimes \alpha_{i_{k}} \otimes \beta_{j_{1}} \otimes \cdots \otimes \beta_{j_{\ell}}$ generates a family of tensors $f_{r, \ell}^{F}: A^{\otimes r} \otimes \beta^{\otimes \ell} \rightarrow \mathbb{C}$, where $f_{r, \ell}^{F}\left(\alpha_{i_{1}} \otimes \cdots \otimes \alpha_{i_{r}} \otimes \beta_{j_{1}} \otimes \cdots \otimes \beta_{j_{\ell}}\right)=c\left(i_{1}, \ldots, i_{r} \mid j_{1}, \ldots, j_{\ell}\right) . \quad$ Put $<x_{i_{1}}, \ldots, x_{i_{r}}, y_{j_{1}}, \ldots, y_{j_{\ell}}>_{F}=f_{r, \ell}^{F}\left(x_{i_{1}} \otimes \cdots \otimes x_{i_{r}} \otimes y_{j_{1}} \otimes \cdots \otimes y_{j_{\ell}}\right)$.

Theorem 5.1. A tensor series $F$ is a Structure Series, iff the collection of tensors $\left.\left\{<x_{1}, \ldots, x_{r}, y_{1}, \ldots, y_{\ell}\right\rangle_{F}\right\}$ forms a System of Disk Correlation Functions. Any System of Disk Correlation Function is generated by a Structure Series.

Proof: Formal calculation demonstrates equivalence the axioms of Disk Correlation Functions and the axioms of Structure Series with the same numbers. 


\section{Noncommutative Frobenius manifolds}

6.1. According to [6], any solution WDVV equation generates a special deformation of Frobenius algebra. In this section we associate to any solution of Structure Equation some deformation of Extended Frobenius algebra.

Definition. Extended Frobenius algebra $\mathcal{H}=\{H=A \dot{+} B,(x, y), x \mapsto$ $\left.x^{*}\right\}$ is a finite dimensional associative algebra $H$ over $\mathbb{K}$ endowed with

a decomposition $H=A \dot{+} B$ of $H$ into a direct sum of vector spaces; an invariant symmetric bilinear form $(x, y): H \otimes H \rightarrow \mathbb{K}$ that is $(x, y)=(y, x)$ and $(x y, z)=(x, y z)$;

an involutive anti-automorphism $H \rightarrow H$, denoted by $x \mapsto x^{*}$;

such that the following axioms hold:

$1^{\circ} A$ is a subalgebra belonging to the centre of algebra $H$;

$2^{\circ} B$ is a two-sided ideal of $H$ (typically noncommutative);

$3^{\circ}$ restrictions $\left.(x, y)\right|_{A}$ and $\left.(x, y)\right|_{B}$ are nondegenerate scalar products on algebras $A$ and $B$ resp.

$4^{\circ}$ an involutive anti-automorphism preserves the decomposition $H=$ $A+B$ and the form $(x, y)$ on $H$, i.e. $A^{*}=A, B^{*}=B,\left(x^{*}, y^{*}\right)=(x, y)$.

A full description of semisimple Extended Frobenius algebras over $\mathbb{C}$ follows from [3] $\S 2$.

The structure tensors $F_{\alpha^{\prime}, \alpha^{\prime \prime}}=\left(\alpha^{\prime}, \alpha^{\prime \prime}\right), F_{\beta^{\prime}, \beta^{\prime \prime}}=\left(\beta^{\prime}, \beta^{\prime \prime}\right), R_{\alpha \beta}=$ $(\alpha, \beta), S_{\alpha^{\prime}, \alpha^{\prime \prime}, \alpha^{\prime \prime \prime}}=\left(\alpha^{\prime} \alpha^{\prime \prime}, \alpha^{\prime \prime \prime}\right), T_{\beta^{\prime} \beta^{\prime \prime} \beta^{\prime \prime \prime}}=\left(\beta^{\prime} \beta^{\prime \prime}, \beta^{\prime \prime \prime}\right), R_{\alpha \beta^{\prime} \beta^{\prime \prime}}=\left(\alpha \beta^{\prime}, \beta^{\prime \prime}\right)$, $I_{\alpha^{\prime} \alpha^{\prime \prime}}=\left(\left(\alpha^{\prime}\right)^{*}, \alpha^{\prime \prime}\right), I_{\beta^{\prime} \beta^{\prime \prime}}=\left(\left(\beta^{\prime}\right)^{*}, \beta^{\prime \prime}\right)$, uniquely describe an Extended Frobenius algebra [3]. Here we denote by $\alpha, \alpha^{\prime}, \alpha^{\prime \prime}$ and $\beta, \beta^{\prime}, \beta^{\prime \prime}$ the elements of the bases $\left\{\alpha_{1}, \ldots, \alpha_{n}\right\} \subset A$ and $\left\{\beta_{1}, \ldots, \beta_{m}\right\} \subset B$ respectively.

6.2. Later we assume that the field $\mathbb{K}$ is the field of real or complex numbers. Associate a deformation of Extended Frobenius algebras to a Structure Series on a space $H=A+B$ with involution $*: H \rightarrow H$ and a basis $\left\{\alpha_{1}, \ldots, \beta_{\ell}\right\} \subset H$. First consider the coordinates on $H$ that associate the element $z=s^{i} \alpha_{i}+t^{j} \beta_{j}$ to collections $\left(s^{1}, \ldots, s^{n} \mid t^{1}, \ldots, t^{m}\right) \in \mathbb{K}^{n+m}$. The correspondences $\alpha_{i} \mapsto s^{i}, \beta_{i} \mapsto t^{i}$ and (tensor multiplication) $\mapsto$ (number multiplication) map tensor series $F\left(\alpha_{1}, \ldots, \beta_{m}\right)$ to formal number series $\hat{F}\left(s^{1}, \ldots, t^{m}\right)$. By definition, put $F_{\alpha_{i} \alpha_{j}}=c(i, j \mid), F_{\beta_{i} \beta_{j}}=c(\mid i, j), I_{\alpha_{i} \alpha_{j}}=F_{\alpha_{i} \alpha_{k}^{*}}, I_{\beta_{i} \beta_{j}}=F_{\beta_{i} \beta_{k}^{*}}$. Let $\left\{F^{\alpha_{i} \alpha_{j}}\right\}$ and $\left\{F^{\beta_{i} \beta_{j}}\right\}$ be the inverse matrix for $\left\{F_{\alpha_{i} \alpha_{j}}\right\}$ and $\left\{F_{\beta_{i} \beta_{j}}\right\}$ respectively. Put

$$
\begin{gathered}
R_{\alpha_{i} \beta_{j}}^{F}=\frac{\partial^{2} F}{\partial \alpha_{i} \partial \beta_{j}}, S_{\alpha_{i} \alpha_{j} \alpha_{k}}^{F}=\frac{\partial^{3} F}{\partial \alpha_{i} \partial \alpha_{j} \partial \alpha_{k}}, \\
T_{\beta_{i} \beta_{j} \beta_{k}}^{F}=\frac{\partial^{3} F}{\partial \beta_{i} \partial \beta_{j} \partial \beta_{k}}, \\
R_{\alpha_{i} \beta_{j} \beta_{k}}^{F}=\frac{\partial^{2} F}{\partial \alpha_{i} \partial \beta_{p}} F^{\beta_{p} \beta_{q}} \frac{\partial F}{\partial \beta_{q} \partial \beta_{j} \partial \beta_{k}} .
\end{gathered}
$$


Theorem 6.1. Let $F$ be a Structure Series. Then at convergence points the tensors $F_{\alpha_{i} \alpha_{j}}, F_{\beta_{i} \beta_{j}}, I_{\alpha_{i} \alpha_{j}}, I_{\beta_{i} \beta_{j}}, \hat{R}_{\alpha_{i} \beta_{j}}=\hat{R}_{\alpha_{i} \beta_{j}}^{F}\left(s^{1}, \ldots, t^{m}\right)$, $\hat{S}_{\alpha_{i} \alpha_{j} \alpha_{k}}=\hat{S}_{\alpha_{i} \alpha_{j} \alpha_{k}}^{F}\left(s^{1}, \ldots, t^{m}\right), \hat{T}_{\beta_{i} \beta_{j} \beta_{k}}=\hat{T}_{\beta_{i} \beta_{j} \beta_{k}}^{F}\left(s^{1}, \ldots, t^{m}\right), \hat{R}_{\alpha^{i} \beta^{j} \beta^{k}}=$ $\hat{R}_{\alpha^{i} \beta^{j} \beta^{k}}^{F}\left(s^{1}, \ldots, t^{m}\right)$ define an Extended Frobenius algebra.

Proof: We shall raise indices of tensors, using the tensors $F^{\alpha_{i} \alpha_{j}}$ and $F^{\beta_{i} \beta_{j}}$. In the case of non-symmetric tensors we always raise the last index. By definition, put $\hat{S}_{\alpha_{i} \alpha_{j} \alpha_{k} \alpha_{\ell}}=\sum_{r} \hat{S}_{\alpha_{i} \alpha_{j}}^{\alpha_{r}} \hat{S}_{\alpha_{r} \alpha_{k} \alpha_{\ell}} \hat{T}_{\beta_{i} \beta_{j} \beta_{k} \beta_{\ell}}=$ $\sum_{r} \hat{T}_{\beta_{i} \beta_{j}}^{\beta_{r}} \hat{T}_{\beta_{r} \beta_{k} \beta_{\ell}}$. It follows from [3] that the above theorem is equivalent to the following conditions:

1) Matrices $F_{\alpha_{i} \alpha_{j}}$ and $F_{\beta_{i} \beta_{j}}$ are nondegenerate;

2) Tensors $\hat{S}_{\alpha_{i} \alpha_{j} \alpha_{k}}$ and $\hat{S}_{\alpha_{i} \alpha_{j} \alpha_{k} \alpha_{\ell}}$ are symmetric with respect to all permutations;

3) Tensors $\hat{T}_{\beta_{i} \beta_{j} \beta_{k}}$ and $\hat{T}_{\beta_{i} \beta_{j} \beta_{k} \beta_{\ell}}$ are symmetric with respect to cyclic permutations;

4) $\hat{R}_{\alpha, \beta_{1}, \beta_{2}}=\hat{R}_{\alpha}^{\beta^{\prime}} \hat{T}_{\beta^{\prime}, \beta_{1}, \beta_{2}}$

5) $\hat{R}_{\beta}^{\alpha^{\prime}} \hat{S}_{\alpha^{\prime}, \alpha_{1}, \alpha_{2}}=\hat{R}_{\alpha_{1}}^{\beta^{\prime}} \hat{R}_{\alpha_{2}}^{\beta^{\prime \prime}} \hat{T}_{\beta^{\prime}, \beta^{\prime \prime}, \beta}$;

6) $\hat{R}_{\alpha, \beta_{1}, \beta_{2}}=\hat{R}_{\alpha, \beta_{2}, \beta_{1}}$

7) $\hat{I}_{\alpha_{1}}^{\alpha^{\prime}} \hat{I}_{\alpha^{\prime}, \alpha_{2}}=F_{\alpha_{1}, \alpha_{2}}, \hat{I}_{\beta_{1}}^{\beta^{\prime}} \hat{I}_{\beta^{\prime}, \beta_{2}}=F_{\beta_{1}, \beta_{2}}$;

8) $\hat{I}_{\alpha_{1}, \alpha_{2}}=\hat{I}_{\alpha_{2}, \alpha_{1}}, \hat{I}_{\beta_{1}, \beta_{2}}=\hat{I}_{\beta_{2}, \beta_{1}}, \hat{I}_{\alpha}^{\alpha^{\prime}} \hat{R}_{\alpha^{\prime}, \beta}=\hat{I}_{\beta}^{\beta^{\prime}} \hat{R}_{\alpha, \beta^{\prime}}$;

9) $\hat{I}_{\alpha_{1}}^{\alpha^{\prime}} \hat{I}_{\alpha_{2}}^{\alpha^{\prime \prime}} \hat{I}_{\alpha_{3}}^{\alpha^{\prime \prime \prime}} \hat{S}_{\alpha^{\prime \prime \prime}, \alpha^{\prime \prime}, \alpha^{\prime}}=\hat{S}_{\alpha_{1}, \alpha_{2}, \alpha_{3}}, \hat{I}_{\beta_{1}}^{\beta^{\prime}} \hat{I}_{\beta_{2}}^{\beta_{2}^{\prime \prime}} \hat{I}_{\beta_{3}}^{\beta^{\prime \prime \prime}} \hat{T}_{\beta^{\prime \prime \prime}, \beta^{\prime \prime}, \beta^{\prime}}=\hat{T}_{\beta_{1}, \beta_{2}, \beta_{3}}$.

All these conditions directly follow from our definition and the axioms of Structure Series.

Thus, Structure Series generate an Extended Frobenius algebra in the points of $H$, where the series from theorem 6.1 converge. This defines a deformation of Extended Frobenius algebra i.e. some analog of Frobenius manifold [6] for (noncommutative) Extended Frobenius algebras.

\section{EXAMPLES}

7.1. For $\operatorname{dim} B=0$ theorems 4.1 and 5.1 get over into theorem III, 4.3 from 9 that claim that Cohomological Field Theory is equivalent to Formal Frobenius manifolds.

Noncommutative Frobenius manifolds with $\operatorname{dim} A=0, \operatorname{dim} B=1$ are Frobenius manifolds of rang 1 . They are described by formal series $F(\beta)[$.

For $\operatorname{dim} A=1, \operatorname{dim} B=1$. Put $\left\langle\alpha^{n}, \beta^{m}>=<\alpha, \ldots, \alpha, \beta, \ldots, \beta>\right.$. Axiom $7^{0}(\S 5)$ demonstrate that all correlators are determined by correlators $\left\langle\alpha^{n}\right\rangle,\left\langle\beta^{m}\right\rangle$ and $\langle\alpha, \beta\rangle$.

Suppose for example that $\langle\alpha, \alpha>=<\alpha, \alpha, \alpha\rangle=<\beta, \beta\rangle=<$ $\beta, \beta, \beta>=1$ and $<\alpha^{m}>=<\beta^{m}>=0$ for $m>3$. Then according to Axiom $7^{0},<\alpha^{3}><\alpha, \beta>=<\beta^{3}><\alpha, \beta><\alpha, \beta>$ and thus $<\alpha, \beta>$ is equal to 0 or 1 . Moreover, Axiom $7^{0}$ gives $<\alpha^{3}><$ 
$\alpha \beta^{2}>=2<\alpha, \beta><\beta^{4}><\alpha, \beta>+2<\alpha, \beta><\beta^{3}><\alpha, \beta^{2}>$ and thus $<\alpha, \beta^{2}>=0$. Analogously we prove that $\left\langle\alpha, \beta^{m}\right\rangle=0$ for $m>1$. On the other hand Axiom $7^{0}$ gives $<\alpha^{3}><\alpha^{2}, \beta>=<$ $\alpha, \beta><\alpha \beta><\alpha, \beta^{3}>+2<\alpha^{2}, \beta><\alpha, \beta><\beta^{3}>$ and thus $<\alpha^{2}, \beta>=0$. Analogously we prove that $<\alpha^{n}, \beta^{m}>=0$ for $n m>1$.

Thus, if the restriction of Structure Series $F$ on $A$ and $B$ is $\frac{1}{2} \alpha^{\otimes 2}+\alpha^{\otimes 3}$ and $\frac{1}{2} \beta^{\otimes 2}+\beta^{\otimes 3}$ then $F=\frac{1}{2} a^{\otimes 2}+a^{\otimes 3}+\frac{1}{2} b^{\otimes 2}+b^{\otimes 3}+\xi \alpha \otimes \beta$, where $\xi \in\{0,1\}$.

7.2. In analogy with 12 any Extended Frobenius algebra $\mathcal{H}=\{H=$ $A \dot{+} B,(.,), *$.$\} with unite 1$ generates some System of Disk Correlation Functions by the rule $<\alpha^{1}, \ldots, \alpha^{s}, \beta^{1}, \ldots, \beta^{t}>=\left(\alpha^{1} \cdots \alpha^{s} \beta^{1} \cdots \beta^{t}, 1\right)$.

7.3. According to 4, a noncommutative Frobenius manifold in our sense is generated by a topological Landay-Ginzburg theory of $B$-type with $2 D$-brane conditions.

\section{REFERENCES}

[1] Alling N.L., Greenleaf N., Foundations of the theory of Klein surfaces / Lecture Notes in Math. N 219 // Berlin-Heideb.-N.Y.:Springer-Verlag.-1971. p.117.

[2] Atiyah M., Topological Quantum Field Theories, Inst. Hautes Etudes Sci. Publ. Math., 68 (1988), 175-186.

[3] Alexeevski A., Natanzon S., Non-commutative extensions of two-dimensional topological field theories and Hurwitz numbers for real algebraic curves (arXiv:math.GT/0202164).

[4] Brunner I., Herbst M., Lerche W., Scheuner B., Landau-Ginzburg realization of open string TFT. hep-th/0305133

[5] Dijkgraaf R., E.Verlinde, H.Verlinde, Nucl Phys. B 352 (1991) 59; Notes on topological string theory and 2D quantum gravity

[6] Dubrovin B., Geometry of $2 D$ topological field theories, in: Lecture Notes in Math., 1620, Springer-Verlag, Berlin (1996), 120-348.

[7] Kontsevich M., Manin Yu., Gromov-Witten classes, quantum cohomology, and enumerative geometry, Commun. Math. Phys. 164, 525-562 (1994).

[8] Lazaroiu C.I., On the structure of open-closed topological field theory in twodimensions, Nucl. Phys. B 603 (2001), 497-530.

[9] Manin Yu., Frobenius manifolds, quantum cohomology, and moduli spaces, American Math. Soc., Colloquium Publication, v.47 (1999).

[10] Natanzon S.M., Moduli spaces of real curves. Moscow Math. Soc., 1980, N 1, $233-272$.

[11] Natanzon S.M., Moduli of real algebraic surfaces, and their superanalogues. Differentials, spinors and Jacobians of real curves, Russian Math. Surveys, 54:6 (1999), 1091-1147.

[12] Natanzon S., Turaev V., Systems of correlators and solutions of the WDVV equations, Commun. Math. Phys. 196 (1998), 399-410.

[13] Witten E., Topological sigma models, Commun. Math. Phys. 118 (1988), 411419 . 
[14] Witten E., On the structure of the topological phase of two-dimensional gravity, Nucl. Phys. B 340 (1990), 281-332.

S.M. Natanzon

Moscow State University

Institute Theoretical and Experimental Physics

Independent University of Moscow

natanzon@mccme.ru 